\title{
Challenges in Modular Learning: Teaching Physical Education in the midst of COVID-19 Pandemic
}

\author{
Jose D. Delas Peñas \\ josedelaspinas@jrmsu.edu.ph \\ Jose Rizal Memorial State University - Tampilisan Campus, Zamboanga del Norte, 7116, Philippines
}

\begin{abstract}
The implementation of Flexible Learning in various educational institutions amidst the COVID-19 pandemic poses many struggles particularly in teaching Physical Education (PE). This study aimed to explore the challenges experienced and encountered by teachers in teaching PE in times of pandemic, particularly during the first semester A.Y. 2020 - 2021 in Jose Rizal Memorial State University Tampilisan Campus wherein Modular Learning Modality (MLM) was implemented. The study utilized a qualitative research design, particularly phenomenology, involving six (6) teachers handling PE subjects. An in-depth interview was employed using an interview protocol that guide the researcher in gathering data. Based on the result of the study, there were three distinct themes emerged, such as; (1) studentsreadiness related challenges, which includes students' readiness, submission of outputs, difficulty in following instructions, and the difficulty of some learning activities and assessment; and (2) teachinglearning process-related challenges which include the lack of preparedness for instruction, difficulty in assessing and providing feedback, and difficulty in establishing the validity of written outputs. Furthermore, an intervention plan was proposed based on the result to address the identified challenges appropriately. Moreover, it was recommended that the proposed intervention be implemented to address issues and concerns in the implementation of Modular Learning, and further studies utilizing other methods, context, or discipline will be conducted.
\end{abstract}

Keywords: Flexible Learning; Modular Learning Modality; Distance Learning; Modular Pedagogy

\section{Introduction}

Coronavirus disease (COVID - 19) has been a very challenging health concern that affects people all around the globe. The major impact of the pandemic is on the education sector from basic education to higher education, since the student cannot be physically present in school due to the implementation of government policies to suspend faceto-face classes in different countries including the Philippines. Furthermore, the PE curriculum aims to promote fitness, health, and wellness by engaging students in different programs and various physical activities, however, with the COVID-19 pandemic, it is quite challenging on the part of the teachers since face-to-face interaction with students is not allowed, and teachers have no choice but to adopt the flexible learning modalities. In Jose Rizal Memorial State University, Flexible Learning System (FLS) was created in response to the call of the Commission on Higher Education (CHED, 2020) to implement flexible learning, and one of the approaches implemented was the Modular Learning Modality.

The implementation of the FL in various educational institutions poses many challenges, particularly on courses that involve various physical activities such as Physical Education (PE) which is best learned through faceto-face encounters. Applying the modular approach in PE is something new, particularly in the JRMSU context. Moreover, considering the concept of continuous improvement, it is important to explore the issues and concerns inherent to its implementation to enable us to see the new realities and look unto this aspect and be able to formulate mechanisms to address them appropriately. Additionally, this study aims to provide evidence-based information, in 
formulating an intervention plan to improve the delivery of instruction using the modular learning approach, particularly in PE.

The COVID-19 pandemic forced colleges and schools to close their doors, affecting an unprecedented number of students around the world (UNESCO, 2019). During the pandemic, it was noted that the academic performance of students was deteriorating, and with the increase of existing socioeconomic disparities affecting learning and outcomes, it can be contemplated that there is growing educational inequities (García \& Weiss, 2017 as cited in Garcia \& Weiss, 2020; United Nations, n.d.). Moreover, due to the pandemic, community quarantine was established resulting in the prohibition of face-to-face classes (Simbulan, 2020). Moreover, CHED (2020) has established guidelines for implementing Flexible Learning (FL) to resume classes for HEIs despite the pandemic, and one of the modalities introduced was modular learning, which was considered as the most preferred mode of instruction by parents for their children (Malipot, 2020), however, there are challenges identified in various literature. Accordingly, modular learning may not work for some students since some of them cannot read and comprehend on their own (Perez, 2020). Other challenges were identified with the implementation of FL are related to preparing the lesson, conduct of classes, internet use, evaluation of students learning, and virtual classroom behavior (Mayol, 2020). Specifically, in modular learning, Dangle \& Sumaoang (2020) have identified some of the challenges encountered by teachers, like students may not able to study independently, late submission of outputs, difficulty in printing and reproduction, the validity of learner's answers in the learning modules, and the bulk of paper works. In teaching PE, there are some of the challenges encountered by physical educators such as; challenges related to the increased numbers of the students in the classroom or large class size (Betram \& Kaleeswaran 2017; Oudat, 2016), challenges of the abilities, devices/instruments, and equipment particularly inadequate trained or lack of competent teachers, lack of facilities, equipment, and materials for teaching (Betram \& Kaleeswaran, 2017; Edward, 2015; Oudat, 2016), challenges related to the content of the academic curriculum, particularly on the quality of instruction of physical education is not delivered and lack of professional preparedness ( Betram \& Kaleeswaran, 2017; Edward, 2015; Oudat, 2016), challenges related to the school environment (Oudat, 2016; ), challenges related to the school management. (Oudat, 2016), time allocation is not sufficient (Betram and Kaleeswaran, 2017), and teachers have a negative attitude towards teaching PE (Edward, 2015). In addition, Betram and Kaleeswaran (2017), noted that the time allocation in the school curriculum for PE has been diminishing around the globe.

Jose Rizal Memorial State University - Tampilisan Campus (JRMSU-TC) is implementing Modular Learning as one of the Flexible Learning Modalities. Based on the guidelines stipulated in the JRMSU FLS Handbook, MLM courses is to be delivered using emails and on-campus pigeon boxes (OCPBs), File Transfer Protocol (FTP), Teacher-Initiated Phone (TIP) calls, and Student-Initiated Phone (SIP) calls which complements the modular learning modality (Committee on Flexible Learning, 2020). Furthermore, in JRMSU - TC, it can be observed that implementing the modular learning approach has been a very challenging experience among the PE teachers, various complaints, and verbalization of inconvenience I have overheard from my colleagues. It can also be noted that at present, there was no known study conducted specifically to the challenges encountered by teachers in teaching PE utilizing the modular learning modality amidst the COVID-19 pandemic. Moreover, the COVID-19 pandemic provides a new perspective in teaching with the implementation of modular learning, and varied experiences among educators especially those who are teaching PE in the JRMSU-TC context prompted me to explore this phenomenon and conducted this study.

Hence, this study was conceptualized to explore the challenges encountered by teachers in teaching Physical Education utilizing a modular learning approach amidst the COVID-19 pandemic which will serve as a basis in developing an intervention plan to improve the teaching-learning process, improve the quality of instruction, and ultimately, to facilitate the attainment of learning outcomes among learners. Generally, the main purpose of this study was to explore the actual challenges encountered and experienced by the teachers in teaching PE amidst the COVID19 pandemic during the first semester A.Y. 2020 - 2021 in Jose Rizal Memorial State University - Tampilisan Campus. Specifically, this study aimed (1) to determine the challenges encountered by teachers in teaching PE utilizing Modular Learning Modality in times of COVID-19 pandemic, and (2) to develop a proposed intervention plan based on the result.

\section{Methodology}

\subsection{Research Design}


This study utilized a qualitative research design, specifically the phenomenology approach. Qualitative research is a systematic empirical inquiry on the meaning or subject of inquiry (Shank, 2002 as cited in Ospina, 2004). Some of the advantages in doing qualitative research are its sensitivity to the contextual factors and increase the opportunity to develop new ideas and theories (Conger, 1998; Bryman et al, 1988; Alvesson, 1996 as cited in Ospina, 2004). Among the qualitative designs, phenomenology was deemed appropriate for this study since it involved descriptive accounts of the personal experience of teachers in teaching PE in a particular phenomenon which is the COVID-19 pandemic. Phenomenology can be used to study the human experience, and structures of one's consciousness as experienced based on the first-person point of view (Smith, 2008 and Sokolowski, 2002 as cited in Gallagher, 2012). Moreover, to describe the challenges encountered by teachers teaching PE in times of pandemic, I took the descriptive account of the participants about the phenomenon based on their responses in the in-depth interview.

\subsection{Research Environment}

The study was conducted specifically in Jose Rizal Memorial State University - Tampilisan Campus (JRMSU - TC), which was situated in Znac, Tampilisan, Zamboanga del Norte. JRMSU - TC is composed of three colleges such as the College of Education, the College Arts and Sciences, and the College of Agriculture and Forestry offering different degree programs. Furthermore, Junior and Senior High School Basic Education Curriculum were also offered which served as laboratory school under the College of Education.

JRMSU system implemented flexible learning modalities such as modular learning and online modalities (JRMSU, 2020). During enrolment, students were asked to select which of the two learning modalities they would prefer whether Online Learning Modality (OLM) or Modular Learning Modality (MLM). As of first-semester A.Y. 2020 - 2021, the student population in JRMSU-TC was 2, 723 of which 181 (7\%) were enrolled in an online learning modality, and 2, 542 (93\%) were enrolled through modular learning modality. The total number of faculty members is 89 , composed of 40 faculty members with permanent status, 19 with temporary status, and 30 visiting lecturers. Among the faculty members, six (6) are handling PE subjects at both secondary and tertiary levels.

\subsection{Participants}

The participants of this study are six faculty members teaching PE. The participants were selected through total enumeration. I believed that the teacher participants of this study have the knowledge and inputs for the completion of this study since they have first-hand information based on their experience on the challenges, they encountered in teaching PE.

\subsection{Instrumentation}

To facilitate the data gathering to answer the research questions, I utilized an interview protocol employing a semi-structured interview. An interview protocol was used to guide me in the conduct of an in-depth interview. An interview protocol is a procedural guide for directing qualitative research through the interview process (Jacob \& Furgerson, 2010).

\subsection{Data Collection Procedures}

The data collection involved a series of events. First, I wrote a letter to the campus administrator asking permission to conduct the research study, and for the administration of the in-depth interview with the faculty members teaching PE on the campus. Then, I talk to the participants one by one to set a schedule for the interview while explaining to them the purpose of the interview and the study. A letter of consent was also prepared to explain to the participants the purpose of the interview and their role in the completion of the study. As the participants agreed to participate, the informed consent was secured by allowing them to affix their signature on the letter of consent. With the agreement of the participants considering their availability and convenience, I set the schedule of the interview.

The interview was done through Google Meet in consideration of the present situation in which face-to-face interactions were discouraged. On the day of the interview, I sent a Google Meet link to the participant, and once again I explained to them the purpose and the mechanics of the interview. Upon agreement, I conducted the interview one by one as scheduled. The interview was recorded since I was using a Google Suite account, and the Tactiq for Google 
Meet Transcription was also used to facilitate recording and documentation. After the interview was transcribed, a copy was sent to the participants for them to review the accuracy of the information gathered. Moreover, the data gathered was treated with utmost confidentiality, and the anonymity of the individual participant was considered throughout the research study.

\subsection{Data Analysis}

Inductive Thematic Analysis was used to analyze the data. Thematic analysis was "a process of identifying patterns or themes within qualitative data" (Maguire \& Delahunt, 2017, p. 3352). Moreover, Colaizzi's descriptive phenomenological method was used in the analysis of data, to reveal the "essence" or "essence structure" of the phenomenon (Morrow, Rodriguez, \& King, 2015). Colaizzi has identified seven steps in the process providing rigorous analysis such as familiarization, identifying significant statements, formulating meanings, clustering themes, developing an exhaustive description, producing the fundamental structure, and seeking verification of the fundamental structure (Morrow, et al.,2015).

Following the steps provided by Colaizzi as presented in Morrow et al. (2015), the data gathered was collated and the transcription was plotted in Microsoft Excel including the interview questions, and the corresponding answers of the different participants. Organizing the data in this process allowed me to be familiar with the data. After I familiarize the data, I identified the significant statements from the data and analyzed them to reveal their meaning or "essence". After which, a code was provided for each statement using color coding since I am using Microsoft Excel to facilitate the identification of different codes, and group them according to categories or themes, and then I clustered the themes that emerged from the analysis and I identified meaning on the different themes common across the narrative accounts. After the clustering of themes, I wrote an inclusive description of the phenomenon, then I condensed the exhaustive description down to a short statement capturing salient aspects, that are essential to the structure of the phenomenon. After analyzing the data, I reviewed the narrative accounts of the participants which served as the fundamental structure of the phenomenon and ensured that the results captured their experiences. Furthermore, in the analysis of data, I ensured that the information provided by the participants are accurate based on the information they have provided, and I also consider that the data was free from bias, neutral, credible, and valid. Moreover, the trustworthiness of the data was ensured considering the credibility of the respondents and information they have provided, dependability of the data gathered using appropriate technology, and confirmability and transferability of the research process by ensuring that the data collection process, the analysis of data, and the interpretation of data follows appropriate process and rigor of research.

\section{Results}

\subsection{Challenges Encountered in Modular Learning Modality}

Two distinct themes emerged based on the result of the study. The following themes were identified: (1) students-readiness-related challenges; and (2) teaching-learning process-related challenges.

\subsubsection{Student-Readiness Related Challenges}

This refers to the challenges encountered by the participants in terms of students' readiness for independent learning, submission of outputs, difficulty in following instructions, and difficulty of some learning activities and assessments. In implementing the Modular learning modality various challenges were encountered by the teacher related to student readiness.

\subsubsection{Lack of Students' Readiness for Independent Learning}

This refers to the challenge encountered by the participants relative to students' readiness to learn independently through modular learning, and lack of readiness to use technology-mediated instruction such as Google classroom. In Modular Learning, students become more independent in learning with less intervention from the teacher. Moreover, participants verbalized their concerns as they encounter challenging experiences in this aspect. Participants verbalized that: 
"The students themselves are not that ready to face this type of teaching, this modular type of learning...Why? Because there are those students who are not doing their tasks in their modules." (Participant 003)

"I think they were not ready yet. They're not yet ready for this approach...this kind of approach ... this modular." (Participant 006)

"I think they are not ready because they are not even familiar even with the Google classroom." (Participant 005)

The result elucidated the importance of students' readiness. The abrupt suspension of face-to-face classes due to the COVID-19 pandemic forced the educational institutions to offer flexible learning, students have no option but to dwell on the available options of learning although assessment on their capability in terms of their mental, psychological, emotional, and other dimensions were not considered to ensure their preparedness in this mode of learning.

\subsubsection{Late Submission of Outputs}

This challenge describes the situation encountered by the participants in terms of students' late submission of outputs since some of them have technical difficulty on submission, and some have taken advantage of the flexibility of time in which the imposition of the deadline was discouraged. Participants reported that:

"We are flexible, meaning we don't need to push or force students to pass the outputs even it is already the deadline...they think that it's okay to pass all the requirements days or weeks before the end of the semester." (Participant 001)

"There are those students who are also very impossible to turn in their outputs, which sometimes I feel like I do not know how to deal with them...some of them will tell you that they already submitted it, and when you look at the classroom (Google Classroom) it is not there." (Participant 004)

"Students cannot pass their outputs in a scheduled time." (Participant 005)

"I asked my students to submit their output on the Google Classroom, however, there were no submissions there." (Participant 006)

This information provided insights on the importance of setting clear guidelines and means of submission within the students' capacity and capability. Outputs were submitted to provide feedback to the teachers on students' progress and to be able to come up with remediation if needed. Furthermore, the data suggested that some students are not prepared, and have a lack of technical competence in using technology such as google classroom for submission of outputs. Submission of output can be considered as an important part of modular learning since it provides the opportunity for the students to present evidence that they have done what is asked of them, and aid teachers in monitoring the learning and progress of students.

\subsubsection{Difficulty in Following Instructions}

This challenge refers to the student's difficulty in following and understanding the instructions, and the inability to submit properly done output. In the FLP, there are various learning activities that the students need to do as it contains all the necessary information for them to read and understand, however, participants have encountered a challenging situation on this aspect. Participants shared that:

"I think there is a problem in understanding the instruction." (Participant 002)

"The problem with the output submitted is that it was not properly done. So, I think it has something to do with the instruction, I don't know if they read instruction or they just choose to read part of the instruction." (Participant 001) 
"It is very challenging when you're trying to give your best so that the student will be able to understand all the instructions on the activities. But then, some students find it very difficult...even though the instructions are very simple." (Participant 004)

The data pointed out the importance of providing instructions based on the students' level of understanding. Instructions are crucial as it guides how certain activities can be done. It can be noted that students may have issues with their literacy skills, such as understanding and following instructions in the learning module. This result can also be linked to the student's preparedness since students are not used to engaging in independent learning wherein, they have to learn with less intervention from the teacher.

\subsubsection{Difficulty of Some Learning Activities}

This refers to the situation in which the participants encountered students complaining about the difficulties of some learning activities in the learning module. Participants noted that there are activities in the learning module that the students find very difficult without the guidance of the teachers. It was quite challenging on the part of the teacher, some of the participants narrated that:

"I know that they (students) also face challenges in dealing with the lessons in the module...because there are lessons which I know that it is not easy for them to understand or to comprehend." (Participant 001)

"There are those difficult activities for the students to do without the guidance of the instructor." (Participant 003)

"There are students who find it very difficult, and they will tell you that it's really hard." (Participant 005)

The result illuminated the importance of designing and developing learning activities that can be done by the general population of students. The result indicated that some of the learning activities may be too difficult for some students, and can be related to a lack of students' readiness to work alone, a lack of comprehension and understanding such as the instructions, and the complexities of the instructions in the learning activities.

\subsubsection{Teaching-Learning Process Related Challenges}

These challenges were encountered by the participants involving the teaching-learning process, such as lack of preparedness for instruction among teachers, difficulty in assessing and providing feedback, and difficulty in establishing the validity of the written outputs. Despite the COVID-19 pandemic, teachers ensured that there was continuity of learning with the implementation of Modular Learning, however, as reported by the participants, there were challenges they have encountered on this aspect.

\subsubsection{Lack of Preparedness for Instruction}

This refers to lack of readiness among teachers to utilize a modular learning approach, lack of preparedness, unavailability of learning modules, and unpolished learning modules containing an error and unrealistic learning activities. The delivery of instruction in PE using MLM was a new experience for the teachers. Participants expressed their concerns on how it should be delivered effectively. Some of the participants conveyed that:

"I was not prepared because I didn't see it coming." (Participant 001)

"The very first thing you need to do as an instructor in physical education, is you need to study your lesson ... with current situations... just rely on the text and then that's it. Just waiting for the outputs of the students that's all... Here in JRMSU, I found out that they don't have the proper manual in PE." (Participant 004)

"I have a hard time with regards to teaching PE because I am not used to having a modular teaching in this subject. We are not used to this and we are still adjusting." (Participant 005) 
Some of the teachers who are non-PE majors also expressed their concern about teaching PE using the MLM. One of the participants remarked that:

"It's very difficult because this is not my major, but I tried my best and then I communicate also with my previous instructor... on what I am going to do?... I ask for a learning module for PE from other institutions I ask here in JRMSU, but they cannot provide yet the module since it is still being prepared, but the class has already started."

(Participant 006)

In another instance, participants also expressed their concern about the preparedness of the learning module to be used in the instruction. One participant articulated that:

"I can see errors on the text, I saw the activities, and I think they are not that realistic."

(Participant 003)

The result indicated that preparedness for instructions in PE considering the teacher and the learning modules, was not that fully in place since some issues were encountered by the participants. Furthermore, the result emphasized the importance of preparation considering the teacher's preparedness and availability of learning resources. It can be understood that there was a time constraint for these aspects, given the bulk of things to be prepared such as the learning materials, from conceptualization, development, finalization, reproduction to distribution, however, it should be given much attention since the quality of instruction is at stake.

\subsubsection{Difficulty in Assessing and Providing Feedback}

This challenge described by the participants a situation in which they cannot see students performing the physical activities, not being able to correct them if they did not perform it correctly, and not being sure if students learned the skills that they want them to learn. Participants expressed their concern about these aspects with the implementation of MLM. Some of the participants mused that:

"And I'm not that sure if they are doing their job or not...the most challenging part of a physical education teacher... there are lots of activities that require the student to do practical exercises, and you cannot see them performing those activities and that's very difficult." (Participant 003)

"Assessing their skills would be quite questionable because I am not sure if they do get the Skills that I wanted them to learn." (Participant 001)

"You cannot directly correct them. When they are performing it right or in the wrong way." (Participant 005)

The importance of assessing and feedbacking as an integral part of the teaching-learning process was pointed out in the result. In teaching PE using a modular approach, it was a challenge since participants cannot see them performing, instead, other forms of evidence such as pictures or videos were asked, however, there were also limitations among students. Furthermore, this implied that modular learning in teaching PE is a challenging task especially in terms of assessment, and in providing feedback. Assessments in PE are mostly performance-based, and a modular approach may not be very effective as PE requires students to demonstrate and perform skills.

\subsubsection{Difficulty in Establishing Validity of Written Outputs}

This refers to the challenge encountered by the participants concerning the reduction of quality of the teaching-learning process, lack of validity of outputs, and the existence of dishonesty such as copying of output of other students. Submission of outputs served as evidence that the students are doing their tasks and learning important concepts as they go over with the modules. However, with the absence of teachers who guide and watch over the students as they do the different activities, the validity, and quality of the student's output would be at stake. This was also a challenge that the participants expressed during the interview. Some of the participants imparted that: 
"Many things were lost during the pandemic, and with the modular approach, the quality of learning was also reduced in our lesson... another challenge is the validity of their performance, I mean the validity of their outputs, I know that it's not 100\% valid and reliable." (Participant 001)

"I'm not that sure if, they are the ones who are doing their output or not." (Participant 003)

"I've seen a lot of copied answers copied written activities from their classmates."

(Participant 006)

In modular learning it is a challenge to ensure the validity of results, especially in written works of students wherein, resources online are available and the student may just copy and paste it on their output without even trying to understand the concepts and worst copying answers from their classmates which is very common. It is somehow a trend among students nowadays which depleted the purpose of various learning activities which supposedly trained them to acquire critical thinking and other higher-order-thinking skills by simply copying from a source without even thinking at all. It is a great challenge for teachers to instill academic honesty among students.

It implicated the importance of establishing the validity of written outputs done by the students. Ensuring the validity of outputs is also ensured the quality of students' learning as it provides them the opportunity to write their outputs utilizing high-order-thinking skills as it operates while they are conceptualizing their written works.

\section{Discussion}

This study explored the challenges encountered by the teachers in teaching PE utilizing MLM amidst the COVID-19 pandemic in the context of JRMSU-TC. The findings indicate that various challenges have been encountered in various aspects which can be categorized into (1) students-readiness-related challenges; and (2) teaching-learning process-related challenges.

\subsection{Students-Readiness Related Challenges}

The findings suggest that the participants believed that students were unprepared in engaging in independent learning through MLM since they are used to relying on teachers' intervention during face-to-face class. The paradigm shift in education as the COVID-19 pandemic strikes forced the educational institutions to shift into flexible learning. With the abrupt changes, students seem to have no option but to dwell on feasible modalities being offered, and ensuring their preparedness in this mode of learning was not put in place. The result contradicted Barrera, Jaminal, and Arcilla (2020) who claimed that students are ready for flexible learning. Moreover, the result was supported in Baticulon et al. (2020) who identified individual barriers in learning during a pandemic such as a difficulty adjusting learning styles, mental health difficulties, physical health issues, and practical concerns. Additionally, Baticulon et al. (2020) also identified technological barriers such as lack of devices or limited access due to gadget sharing, unreliable, slow, or no internet access, lack of technical skills, and issues with the online learning platform. Furthermore, according to Parkes, Stein, and Reading (2015), students were unprepared for a range of e-learning competencies, particularly in some aspects such as time management, critical thinking skills, and collaborating with others.

With MLM, students seemed to delay submissions of outputs banking on the premise of flexible learning. Moreover, the findings suggest that some students have to lack technical competence in using technology such as google classroom for submission of outputs which contributes to the delay. This was consistent in Dangle \& Sumaoang (2020) which stressed that submission of outputs was often late and most of the learning activities were returned unanswered. However, the result provided insights on the importance of setting clear guidelines and means of submission within the students' capacity and capability

Another important insight uncovered in the result is the crucial role of giving instructions and the level of difficulty of learning activities in the learning modules. Students seem to have issues in understanding and following instructions, and the learning activities were complex and seems to be too difficult for them since they will be working alone. These can link to their lack of readiness in MLM, as it engages them independently with fewer teachers' interventions. The result supported the report in Perez (2020) that learners especially in a remote area cannot read and comprehend on their own, hence, modular learning may not work in some areas in the country. Furthermore, Dangle and Sumaoang (2020) also pointed out that learners cannot study independently, and reported that $70 \%$ of the learners cannot follow instructions in the learning module. Furthermore, this is also supported in De Villa and Manalo (2020) 
who stated that one of the challenges encountered by teachers was the complexity of the assessment. Furthermore, the study in Dangle \& Sumaoang (2020) also found out that $90 \%$ of the participants in their study are having a hard time answering their modules. Moreover, the result highlights the importance of providing clear instructions and designing learning activities based on students' level of understanding, capacity, and capability.

\subsection{Teaching-Learning Process Related Challenges}

In terms of readiness, teachers are not an excuse in implementing the MLM since they are the ones who are preparing the learning modules. However, the limited amount of time for preparing the learning materials has brought concern for its implementation. The result suggests that preparedness for instructions in PE considering the teacher and the learning modules was not that fully in place. This confirmed the report in Mayol (2020) which pointed out that one of the challenges in teaching amidst the COVID-19 pandemic was the preparation of the lesson which is different from what the teachers used to in the past. Furthermore, De Villa and Manalo (2020) also affirmed that another challenge encountered by teachers was the difficulty in instructional delivery since they have limited skills and knowledge on ICT. Similarly, Inan (2021) also confirmed that educators are having technical and educational difficulties in conducting distance learning.

Assessment and providing feedback are an integral part of the teaching-learning process. With MLM, this seems to be very challenging since the teachers cannot personally witness the performance of students in various physical activities. In PE, assessment is mostly performance-based which requires students to demonstrate and perform skills to support the attainment of learning outcomes. Alternatively, since there are no face-to-face classes, other forms of evidence such as pictures or videos may be used, however, there are also limitations among students in terms of access to technologies, thus, led to delay submission and even non-submission at all which may result to another challenge which is the giving of feedback to students' output. This was supported by Jeong and So (2020) who found out that although students could submit physical education performance evaluations in the form of videos and written assignments, it is very time-consuming. Furthermore, the result was also consistent in Estrada (2021) which stated that there is limited or no feedback at all in Modular Learning. Additionally, Estrada (2021) also pointed out that modular learning has become an endless stream of paperwork for teachers and students, and less attention is given to its effectiveness. In addition, Dangle and Sumaoang (2020) also reported that the bulk of paper works, and papers to check and record add up to the challenges encountered by teachers in the new normal.

Another challenge encountered by teachers related to assessment is the validity of the written outputs of students. Technology nowadays offers easy access among students to various information from the internet which they can use just to accomplish their tasks. This is supported by Yilmaz (2017) who found out that the cheating problem remains the main problem faced in online exams. Similar problems also occur in the assignment and project in which students copied and pasted things they found on the internet or copied one another's work. Additionally, Dangle and Sumaoang (2020) also affirmed that some teachers are reluctant on the learner's answers on the learning module in terms of its validity, and the probability that the students have mastered their lesson. Nevertheless, the validity of outputs ensured the quality of students' learning as it provides them the opportunity to write their outputs utilizing high-order-thinking skills as it operates while they are conceptualizing their written works.

\subsection{Proposed Intervention Plan}

Based on the result of the study on the challenges encountered by teachers in teachings Physical Education in times of COVID-19 Pandemic, an intervention plan can be designed. Designing a proposed intervention plan was the ultimate goal of this study to improve the teaching process despite the present situation. Integrating the findings of this study, the areas of concerns to be addressed in this intervention plan are as follows: difficulty in communication, lack of access to the internet and limited access to technology, lack of student's readiness, late submission of outputs, difficulty in followings instructions, the difficulty of some learning activities, lack of preparedness for instruction, difficulty in assessing and providing feedback, and difficulty in establishing the validity of written output. The proposed intervention plan aims to achieve the following: (1) identify appropriate interventions to address the different challenges encountered by teachers in teaching PE in times of COVID-19 pandemic; and (2) improve the delivery of instruction in Physical Education particularly in this time of COVID-19 Pandemic through the implementation of various actions that address different issues in the implementation of modular learning modality in PE. 
To specifically address the issues and concerns identified in this study, there are some of the actions which can be considered in the proposed intervention plan. To address the lack of students' readiness for independent learning, orientation should be conducted to ensure the readiness of students on various aspects of how the teachinglearning process be implemented utilizing the modular learning approach, and assessment of students' readiness should be carried out to guide the university in formulating policies and designing learning resources that matched with students' capacity and capability. Moreover, to address late submission of outputs, the importance of the submission of outputs should be clearly emphasized during class orientation, and students should be informed regarding the purpose of the submission which is part of the formative assessment. Orientation may be conducted utilizing prerecorded video presentation or any other means and made available and accessible to students. In addition, students should be equipped with the technical competence in using technology for submission, and be informed of other means of submission. FLS Guidelines on the distribution and collection of learning resources through electronic mail, and through the Municipal Postal Boxes should be reinforced. To address the difficulty in following instructions, and the difficulty of some learning activities, clear guidelines for the development of learning modules particularly in designing appropriate activities and instructions should be in place and should be integrated as criteria for evaluation during an internal review of the learning modules or the Flexible Learning Package. Complex instructions should be broken down into simplified form through the development of specific instructions which is the step-by-step procedures on how each activity be done using simple terms based on the student's level of understanding.

To address the lack of preparedness for instruction, planning should be properly done by assigning faculty members in the development of learning modules, and strict monitoring should be done to ensure that the materials are available at the start of classes. This also entails management strategies to motivate the teachers to pursue the development of modules for the sake of all learners. Furthermore, equipping teachers' competence through capacitybuilding activities should be done, considering a holistic approach to prepare them, physically, mentally, and emotionally to engage in new modalities of teaching, and providing the necessary assistance in terms of technical aspects and providing the appropriate equipment to facilitate compliance. To address the difficulty in assessing and providing feedback, guidelines on assessment and feedbacking specifically designed for PE should be in place with the consultation of stakeholders, especially with the parents, LGUs, and other agencies to ensure the safety of students and teachers should be in place. Lastly, to address the difficulty in establishing the validity of written outputs, the FLS guidelines in ensuring academic honesty in all learning activities should be reinforced and implemented appropriately.

\section{Conclusion}

The narrative accounts of the participants on the actual challenges they have encountered and experienced in teaching PE utilizing Modular Learning Modality in times of COVID-19 pandemic provided insights and identified challenges from the multiple facets of their experience with the phenomenon. The exploration revealed two general themes that emerged which include, students-readiness related challenges, and teaching-learning process-related challenges.

The study provided evidence that indeed, Modular Learning Modality used in teaching Physical Education when face-to-face interaction between students and teachers was prohibited was a challenging experienced. Challenges concerning various aspects of the teaching-learning process were experienced by the participants which should be addressed accordingly as it may affect the learning experiences of students and the attainment of learning outcomes.

The teaching experience of the participants amidst the COVID-19 pandemic, specifically in PE, marks a significant milestone in terms of pedagogy in PE as it revealed various limitations and difficulties of Modular Learning Modality, concerning the readiness of students, and teachers, and the preparedness of learning resources. Furthermore, it can be concluded, that implementing the Modular Learning Modality in teaching PE in the JRMSU-TC context does not work smoothly and efficiently enough with the identified challenges that also mark the avenues that need improvement. Thus, an intervention plan was proposed and should be implemented to address them appropriately.

\section{References}

Barela, M., Dionisio, J., Heimerl, K., Sapitula, M., and Festin, C. (2019, January 9). Deploying community cellular networks in rural barangays in the Philippines. Association for Progressive Communications. 
https://rising.globalvoices.org/blog/2019/01/09/deploying-community-cellular-networks-in-ruralbarangays-in-the-philippines/.

Barreara, K., Jaminal, B., \& Arcilla, F. (2020). Readiness for flexible learning amidst COVID-19 Pandemic of Saint Michael College of Caraga, Philippines. SMCC Teacher Education Journal (2) 1 - 15. https://www.researchgate.net/publication/344374652_Readiness_for_Flexible_Learning_amidst_COVID19_Pandemic_of_Saint_Michael_College_of_Caraga_Philippines.

Baticulon,R., Alberto, N., Barron, M., Mabulay, R., Rizada, L., Sy, J., Tiu, C, Clarion,C., \& Reyes. J. (2020). Barriers to online learning in a time of COVID-19: a national survey of medical students in the Philippines. medRxiv. https://doi.org/10.1101/2020.07.16.20155747.

Betram, J. \& Kaleeswaran, P. (2017). Challenges in physical education and sports sciences - A comparative study. International Journal of Interdisciplinary Research in Arts and Humanities, 2 (2). 107-108.

Commission on Higher Education (2020). Guidelines on the implementation of flexible learning. CMO No. 04.https://ched.gov.ph/wp-content/uploads/CMO-No.-4-s.-2020-Guidelines-on-the-Implementation-ofFlexible-Learning.pdf

Committee on Flexible Learning (2020). Jose Rizal Memorial State University: Flexible Learning System (Learning Continuity Plan). Jose Rizal Memorial State University. Philippines.

Committee on Flexible Learning (2020). Jose Rizal Memorial State University: Flexible Learning Handbook. Jose Rizal Memorial State University. Philippines.

Dangle, Y. \& Sumaoang, J. (2020). The implementation of modular distance learning in the Philippine secondary public schools. 3rd International Conference on Advanced Research in Teaching and Education. https://www.dpublication.com/wp-content/uploads/2020/11/27-427.pdf.

De Villa, J. \& Manalo, F. (2020). Secondary teachers' preparation, challenges, and coping mechanism in the preimplementation of distance learning in the new normal. OER International Multidisciplinary Research Journal. 2 (3). 144 - 154. https://papers.ssrn.com/sol3/papers.cfm?abstract_id=3717608.

Edward, S. (2015). Challenges facing the teaching and learning of physical education in primary schools in Bwiri/Nanguba Zone, Samia Sub-County, Kenya. [Unpublished Thesis]. Kenyatta University. https://irlibrary.ku.ac.ke/bitstream/handle/123456789/13467/Challenges\%20facing\%20the\%20teaching\%20and\%20 learning $\% 20$ of $\% 20$ physical\%20ducation $\% 20$ in $\% 20$ primary $\% 20$ schools $\% 20$ in $\% 20 \mathrm{Bw}$ iri $\% 20$ Nanguba $\% 2$ 0Zone, $\% 20$ Samia\%20Sub-County, \%20Kenya.pdf;sequence=1

Estrada, L. (2021, January 12). Are self-learning modules effective? [News Article]. Rappler. https://www.rappler.com/voices/imho/opinion-are-self-learning-modules-effective.

Gallagher S. (2012) What Is Phenomenology? In: Phenomenology. Palgrave Philosophy Today. Palgrave Macmillan, London. https://doi.org/10.1057/9781137283801_2

Garcia, E. \& Weiss, E. (2020, September 10). COVID-19 and student performance, equity, and U.S. education policy [Article]. Economic Policy Institute. https://www.epi.org/publication/the-consequences-of-theCOVID-19-pandemic-for-education-performance-and-equity-in-the-united-states-what-can-we-learn-frompre-pandemic-research-to-inform-relief-recovery-and-rebuilding/

Inan, H. Z. (2021). Challenges of Distance/Online and Face-to-Face Education in the New Normal: Experiences of Reggio Emilia-Inspired Early Childhood Educators in Turkey. Pedagogical Research, 6(1), em0086. https://doi.org/10.29333/pr/9304

Jacob, S.A, \& Furgerson, S.P., (2010). Writing Interview Protocols and Conducting Interviews: Tips for Students New to the Field of Qualitative Research. The Qualitative Report. Volume 17, T\& L Art. 6, 1-10. Retrieved from https://files.eric.ed.gov/fulltext/EJ990034.pdf

Khan, A., Khan, S., Islam, S. \& Khan, M. (2017). Communication skills of a teacher and its role in the development of the students' academic success. Journal of Education and Practice. 8 (1). 18 - 21. https://files.eric.ed.gov/fulltext/EJ1131770.pdf.

Maguire, M., Delahunt, B. (2017). Doing a Thematic Analysis: A Practical, Step-by-step Guide for Learning and Teaching Scholars. All Ireland Journal of Teaching and Learning in Higher Education (AISHE-J). 8 (3). $3351-33514$.

Malipot, M. (2020, August 4). Teachers air problems on modular learning system [News Article]. Manila Bulletin. https://mb.com.ph/2020/08/04/teachers-air-problems-on-modular-learning-system/. 
Mayol, P. (2020, October 5). The paradigm shifts for teachers' challenges in the new normal [Blog Article].

Cebufinest. https://cebufinest.com/paradigm-shift-teachers-challenges-new-normal/.

Morrow, R., Rodriguez, A. and King, N. (2015). Colaizzi's descriptive phenomenological method. The Psychologist, 28(8), 643-644.

Niñal, R. (2020, June 16). Gov't to hand over radios to students in far-flung areas [News Article]. Presidential Communications Operations Office. Republic of the Philippines. https://pcoo.gov.ph/news_releases/govtto-hand-over-radios-to-students-in-far-flung-areas/.

Ospina, S. (2004). Qualitative research. Encyclopedia of Leadership. SAGE Publications. https://ualr.edu/interdisciplinary/files/2010/03/Qualitative_Research.pdf

Oudat, M. (2016). Challenges facing physical education teachers in Jordan from perspective of the teachers themselves. Scientific Research Publishing Inc. https://www.researchgate.net/publication/301740155_Challenges_Facing_Physical_Education_Teachers_i n_Jordan_from_Perspective_of_the_Teachers_Themselves.

Parkes, M., Stein, S., \& Reading, C. (2015). Student preparedness for university e-learning environments. The Internet and Higher Education, 25, 1-10.

https://www.researchgate.net/publication/268691014_Student_preparedness_for_university_elearning_environments

Perez, A. (2020, July 20). Weighing modular learning [News Article]. Sunstar Publishing Inc. https://www.sunstar.com.ph/article/1864236/Davao/Local-News/Weighing-modular-learning.

Simbulan, N. (2020, June 4). The Philippines: COVID-19 and its impact on higher education in the Philippines. The Head Foundation. https://headfoundation.org/HESB8/COVID-19-and-its-impact-on-higher-education-inthe-philippines/

Tria, J. Z. (2020). The COVID-19 pandemic through the lens of education in the Philippines: The new normal. International Journal of Pedagogical Development and Lifelong Learning, 1(1), ep2001. https://doi.org/10.30935/ijpdll/8311

UNESCO. (2019). How are you learning the COVID-19 pandemic? https://en.unesco.org/COVID19/educationresponse/learningneverstops

United Nation (n.d.). COVID-19 and higher education: Education and science as a vaccine for the pandemic. UN. Org. https://www.un.org/en/academic-impact/COVID-19-and-higher-education-education-and-sciencevaccine-pandemic.

Wanyama, M. (2011). The challenges of teaching Physical Education: Juxtaposing the experiences of physical education teachers in Kenya and Victoria (Australia) [Thesis]. The University of Melbourne. https://minervaaccess.unimelb.edu.au/bitstream/handle/11343/36155/271035_challenges+of+teaching+pe+\%28thesis\%29 $+\% 282 \% 29 \% 5 \mathrm{~B} 1 \% 5 \mathrm{D}$.pdf?sequence $=1$.

Yilmaz, R. (2017). Problems experience in evaluating success and performance in distance education: A case study. Turkish Online Journal of Distance Education-TOJDE. 18 (1). 39 - 51. https://files.eric.ed.gov/fulltext/EJ1124961.pdf. 